\title{
PROVEN AND RELIABLE METHODS OF EARLY SPEECH THERAPY INTERVENTION FOR CHILDREN WITH AUTISM SPECTRUM DISORDER
}

DOI: $10.24234 /$ se.2021.3.1.260

\begin{abstract}
AUTHORS' DATA:
Kristina Kharatyan, Speech and Language Therapist

“ARBES" Health care center, rehabilitation branch of "Arabkir" joint medical center \& institute of child and adolescent health, Armenia

Contacts: kristlog@mail.ru
\end{abstract}

Gohar Hovyan, $\mathrm{PhD}$ in Education, Associate professor

Chair of Speech and Rehabilitative Therapy

Khachatur Abovyan Armenian State Pedagogical University, Armenia

Contacts: gohar.hovyan@mail.ru

\begin{abstract}
The purpose of our research is to identify the effective tools for early Speech therapy intervention in working with children with Autism Spectrum Disorder (ASD) in order to provide their speech forming and development by using confinable methods. Besides, the purpose includes the thesis about high effectivity of systematic complex approach in case of work with ASD.

Current research is based on theoretical materials from professional literature and on 14 years of own personal speech therapy professional experience.

It is concluded that methods used in the speech therapy work with ASD children are effective in application, but as a result of the individual approach of the child's speech and communication, their combination at the appropriate stage of development provides a higher result.

We emphasize the development of established methods and means of early speech intervention in the work with ASD, the development of a systemic approach and the introduction of conditions that will facilitate their early speech therapy intervention.
\end{abstract}


Keywords: Autism Spectrum Disorder, communication, gesture, sensory integration, Denver model, the Affolter's approach, ABA, AAC, DTT, PRT, CBT, "Floor time".

\section{LITERATURE REVIEW}

Studies of the professional literature show that there are currently many methods, tools, programs aimed at developing the mental processes, communication, emotional sphere of a child with autism. Based on the results of our professional activities, we can state that almost every existing and widely used method provides the best efficiency due to the combination of individual approaches and means. For example, the leading role in working with children with ASD have approaches, which are meant to correct behavior, and then come the methods of influencing general perception, emotional spheres, and after it- the approaches of communication and speech development.

Today there are two psychological-pedagogical methodological groups - basic and auxiliary.

Basic methods allow the child to master household, communication, working, educational, and professional skills, while auxiliary tools create conditions for the implementation of basic methods (sensory integration, game therapy, art therapy, hypo therapy, sand therapy, Dolphin therapy, swimming, etc).

Nowadays Zwaigenbaum`s and colleagues research in the special edition of "Pediatrics" periodical proves the reliability of the thesis, that it is possible to diagnose ASD at the age below two. This fact more than ever shows the necessity of correction programs for the children with ASD. At the same time, professional intervention for children younger than 2 and 3 years should include developmental methods, so such intervention should occur as soon as possible and base on the study of the child's individual capabilities and difficulties (Zwaigenbaum et al., 2005).

The intervention of the ASD problem should be aimed at consolidating all interests, involved in the problem: family, relatives, kindergarten, social and medical resources, etc. Early diagnosis of ASD is the key to early intervention, which according to a number of studies has a significant impact on the "long-term" positive results for the children with ASD (Morozov, 2002).

Early signs of autism often appear at 18 months or earlier (Woods \& Wetherby, 2003). 
Appropriate intervention should begin with a detailed study of the child's strengths and needs. A clear diagnosis is based on systematic observations, survey of the child's communication, social interaction, behavioral and development level.

\section{AUTHORS NOTES}

According to the World Health Organization's (WHO) report (April 2017), every 160th child in the world has ASD. According to our ten-year (2007-2017) research in Armenia, there was a fivefold increase in the number of children diagnosed with ASD (Kharatyan \& Hovyan, 2019).

This sharp increase makes the interpretation of effective approaches to speech therapy intervention in children with ASD even more important.

During our long-term speech therapy practice (14 years, average number of children peryear - 50), children diagnosed at the age of 0-3 years and included in the development intervention process, registered higher degree of development in the same period and with the same methodological approach, than children older than 3-6 years old with the same diagnosis.

However, according to our research, in Armenia parents first apply for examination and diagnostics for their children at the age of 3 to 6 . In $57 \%$ cases those are boys and in $25 \%$ cases are girls. While talking about early diagnosis at the age of 0-3-year boys make up 15\%, girls-3\%.

\section{MODELS OF SCIENTIFICALLY SUBSTANTIATED EARLY SPEECH THERAPY INTERVENTION}

\section{Communication.}

Social skills training. Social communication activities are called to encourage socially acceptable behavior of the children with ASD and to use social groups and other platforms to teach them peer-to-peer interaction skills, as social communication deficits are a major problem for people with ASD. Research on corrective methods through social communication is still ongoing today (Kimhi, 2014). According to our observations, this method is effective in methodical combined work. 
Joint attention, symbolic play, engagement management. This method involves a combination of developmental and behavioral characteristics. It is based on the basis of social communication (joint attention, play) and includes natural strategies of increasing the speed and complexity of social communication. This approach assumes the joint work of teachers and parents, which is focused on child's different everyday activities in different areas of life (Pierce, et al., 2011).

Game therapy. This method is actively used in the work with children with ASD, as their main independent play activity is the manipulation of toys or non-toy objects (belts, toy parts), which becomes stereotypical and has a self-stimulating nature. In the development process, the game goes through various stages, from simple manipulations to complex symbolic games (Tyulina, 2013). Children with ASD are mainly in the manipulative stage of the game, there may be noticed some elements of functional play, and the symbolic stage of play is particularly affected. Role play is the highest stage of game development, the development of which is associated with the possibility of further socialization of the child, and for most children with autism it is almost inaccessible at the moment when the developmental interventions begin (Greenspan \& Weeder, 2013).

Either the play therapy is ancillary or basic, it is one of the most promising ways to work with children with ASD. In practice, it is more affordable, as it can be used by both professionals and relatives of a child with ASD. The child's family can use play therapy every day, making it the main basis of communication. From our point of view, the attractiveness of play therapy lies in its psychological comfort for the child and the whole family (Woods \& Wetherby, 2003; Ayres \& Dubuque 1974).

Augmentative and Alternative Communication (AAC) can help to complete and compensate for the lack of expressive communication, as many children with ASD have functional speech impairment. This method involves the use of more difficult techniques, from sign language to image systems, such as the AAC image sharing system (Bondy \& Frost, 2001), which is a communication strategy for people with speech impairments and allows to communicate with peers at an early age. It does not teach oral speech at once, but helps to speak with the help of pictures, which further promotes the formation of word-speech and the development of social communication initiatives. Experimental studies have shown the effective use of this method, 
especially when it is used within the framework of the ABA direction. This thesis shows and proves the essence of systematic approach.

Sign Language. In science the issue of gesture-using is still controversial, even for people with hearing impairments. It has been proven that children with autism can be taught sign language as an alternative means of communication; there is evidence that some children have started speaking sign language. Our research has shown that children who have overcome this stage of body language communication are more likely to start speaking and have more meaningful speech.

Visual support. Children with autism have difficulty understanding speech and do not orient themselves in the sequence of actions. So it is very effective to organize the teaching of the mentioned sequence through visual images.

Modeling. This method is especially effective when the work process is videotaped and it is possible to watch it after finishing the work. This method is also applicable to the child's relatives, so it is important, because the intervention carried out by parents is a key element in the stages of preparation for the learning and communication process of children with ASD.

Denver model. The method is based on active therapy performed by children and adults, which is carried out through games that develop communication skills. The method includes peerto-peer interaction in the school environment and homework (Rogers \& Dawson, 2009).

The early intervention model for nursing children is a continuation of the Denver model, which incorporates developmental approaches to behavioral learning strategies that can be implemented in a variety of settings (for example, by a specialist or parent in group or individual classes at home or in a hospital) (Rogers \& Dawson, 2009).

Social stories. In this method a hero is chosen and every day a symbolic story is told to the child about the hero. Applying the method separately is not effective.

Adaptation with peers. Recent studies have shown that when children with autism are in the company of normally developing children, it has a positive effect on their personality development and communication processes. We especially use pedagogical programs, as autism is a permanent impediment to the development of the child, so multifaceted development is needed. Studies show that the methods and programs that keep the child in the daily routine are more effective (Rogers \& Dawson, 2009). 


\section{Behavioral approach.}

Applied Behavioral Analysis (ABA). The method is defined as the application of behavioral principles aimed at modifying specific behavior and evaluating the effectiveness of the intervention. It prevents problem behavior. The use of this method is based on a person's needs, interests and marital status. The ABA method is often used in early professional intervention programs (under 4 years) to develop a child`s necessary and important life skills. Studies show that children with ASD show more positive results if the method is used before the age of 5. ABA has been the basis for a number of other methods, such as Discrete Trial Training (DTT), Pivotal Response Treatment (PRT) and Cognitive-Behavioral Therapy (CBT).

Cognitive-Behavioral Therapy (CBT). The method involves a combination of cognitivebehavioral learning principles aimed at promoting and encouraging desired behavior. The approach is based on the idea that a change in a person's mindset or cognitive patterns can lead to a change in a person's behavior. Various studies have described this method as a way for children with ASD to manage their own behavior and to exhibit appropriate behavior in different situations and environments.

Social thinking means supporting children with pre-school-age ASD, as well as adults with social learning problems (social communication disorders, and similar problems). It includes strategies for teaching pragmatic speech, social emotion, social perception, and skill development. CBT teaches people to understand the logic of the processes taking place (Leaf \& Macken, 2016).

Improving the quality of life for a child with ASD requires the support and involvement of people around him/her, such as social, medical and educational institutions will help control the child with the disorder in his/her daily life.

The Greenspan Floor-time Approach, which is also known as "Floor time" considers working on the floor. Means of communication are formed gradually. The method includes a 5-6hour lesson in two parts, during which the child is taught basic skills on the floor. This method is often combined with behavioral therapy (Greenspan \& Weeder, 2013).

\section{Sensory integration.}

According to the study by American speech therapist and psychologist Jane Ayres, 70\% of children with behavioral, developmental, speech, motor, or emotional disorders have disorders of various sensory systems. As we all know, no part of the human nervous system works separately. In the case of sensory dysfunction, the child is unable to concentrate, which is especially noticeable 
during speech perception, when the brain is unable to analyze large amounts of audio material. Sensory integration is a process in which sensations from different sensory systems are perceived and developed (visual, auditory, taste, smell, touch, proprioceptive, vestibular), which activation is the basis of methodological exercises (Ayres \& Dubuque 1974). This method, however, loses its effectiveness when used as a stand-alone method. It is also necessary to make it an integral part of the correction program.

The Affolter's approach. Based on sensory integration, authored by Swiss researcher Felicie Affolter (Affolter \& Walter, 2020). The essence of the method is to give the child tactile information with support of an adult, which helps the child to perceive and integrate new information and then act. The approach mentions the leading role of the sensory information that comes through the tactile system, which according to Affolter, plays a central role in development, helping the child to think and then act. Due to the integration of the other senses in this process, the method allows the child with ASD to develop speech perception and communication skills at a certain stage, including ergo-therapy in speech therapy (Affolter \& Walter, 2020).

Thus, if in the past in psychological-medical-pedagogical correctional work for each child with autism it was necessary to choose one of the existing methodological approaches as the main one, recently the authors and followers of different approaches do not consider their method as the only correct one. Nowadays is underlined the importance ofs the involvement of parents, in particular, in organizing activities so that they can be continuously implemented at home (Rogers \& Dawson, 2010).

Early and complex intervention significantly affects the quality of ASD childs life, bringing long-lasting and positive changes. As an example we can consider the results of the speech therapy examination conducted in our Center, based on the data of 2007-2008. As an option we studied 16 children with ASD, who used the Center's speech therapy service. The children were divided into 4 groups in the study, four children in each group. The following professional methods were used:

1. First group - the Affolter's approach.

2. Second group - the ABA approach.

3. Third group - game therapy.

4. Fourth group - complex application of methods. 
9 months of observation in all groups shows positive dynamics in the process of speech perception and reproduction, however, the highest efficiency was recorded in 4th group, when the choice and use of methods were periodically changed based on the child's current level of development. Besides, the children of the first 3 groups, where only one of the current approaches was applied, observed a decrease in positive dynamics from the 3rd trimester.

In particular, in the first trimester, the children of all the observed groups had positive dynamics of speech perception and pronunciation, however, in the second trimester, the speed of positive tendency in the first three groups significantly decreased, and in the third trimesterstopped. At the same time, in the fourth group, using complex methods and high results were maintained until the end of the course.

As a result, on the initial stages, the method chosen according to the primary assessment, no matter how appropriate it is to the child's developmental characteristics, provides an effective start, however, in the absence of methodological combination, it does not provide high efficiency.

For example, the ABA method chosen for 2nd group was expedient because the children had behavioral difficulties. However, after the correction of behavior, due to the difficulties of sensory perception, the positive dynamics ceased. At the same time the use of ABA was discontinued when working with children in the 4th group, it was replaced by the Affolter's approach, after which it became possible to use play therapy, and later return to the use of the ABA.

A similar difficulty was observed in the work with the children of the 1st group, when based on the results of the primary assessment, the overcoming problems of the children's emotional sphere became more difficult and the Affolter`s approach was preferred. Later there were some difficulties in the conditions of narrow methodical application. In the case of the 4th group children, with whom the work was initially started by the Affolter`s approach, after overcoming some of the emotional problems, they were easily included in the game therapy.

Thus, it can be concluded that all the methods used in the speech therapy work with ASD children are effective in application, but as a result of the individual approach of the child's speech and communication, their combination at the appropriate stage of development provides a higher result.

We underline the importance of using complex program, as we will have the expected result only in the combination of methods. Of course, this combination must be systematic, necessarily 
carried out with an individual approach to each child, taking into account the child's abilities, needs and specific features his/her study.

Examining, comparing and analyzing the many methods and programs aimed at developing the means of speech communication of children with ASD, we can state that not excluding the possibilities of using modern methods for speech development, it is necessary to emphasize the development of complex methods, their systemic approach and conditions which will facilitate early speech therapy in children with ASD.

\section{REFERENCE LIST}

1. Affolter, F., \& Walter, S. (2020). Tactual Social Interaction: I Feel the Others and Interact with Them, February 26, 21 The Development of Language, 8th Edition.

2. Ayres, J., \& Dubuque, I., A. (1974). The development of sensory integrative theory and practice, Kendall Hunt

3. Bondy, A., \& Frost, L. (2001). The Picture Exchange Communication System. Research Article Find in PubMed https://doi.org/10.1177/0145445501255004

4. Greenspan, S., \& Weeder, M. (2013). On you with autism: using the FLOORTIME technique for the development of relationships, communication and thinking.

5. Kharatyan, K., A., \& Hovyan, R., R. (2019). Problems of early intervention of children with autism spectrum disorders in Armenia, in particular, in the "ArBeS health center" and speech therapy intervention models, Collection of articles "Science and education" part 2 (p.181-184), ICNS “Science and education”, Penza.

6. Kimhi, Y. (2014). Theory of Mind Abilities and Deficits in Autism Spectrum Disorders, Top Lang Disorders, Vol. 34, No. 4, pp. 329 - 343.

7. Leaf, R., \& Macken, D. (2016). Work in progress/Behavioral strategies curriculum for intensive behavioral therapy in autism.

8. Morozov, S., A. (2002). Children's autism and the basics of its correction (materials for the special course). - M.: Publishing house Signal.

9. Pierce, K., Carter, C., Weinfeld, M., Desmond, J., Hazin, R., Bjork, R., \& Gallagher, B. (2011). Detecting, studying, and treating autism early: The one-year well-baby check-up approach. The Journal of Pediatrics, 159 (3), N.11. 
10. Rogers, S., J., \& Dawson, G. (2009). Play and engagement in early autism: The Early Start Denver Model. Volume I: The Treatment, New York, NY: Guilford Press.

11. Rogers, S. J., \& Dawson, G. (2010). Early Start Denver Model for young children with autism: Promoting language, learning, and engagement.

12. Tyulina, V., B. (2013). Game therapy in correctional work with special kids // Organization of correctional and pedagogical action in a special educational environment, materials of the All-Russian scientific-practical conference with international participation.

13. Woods, J., J., \& Wetherby, A., M. (2003). Early identification and intervention for infants and toddlers who are at risk for autism spectrum disorder. Language, Speech, and Hearing Services in Schools.

14. Zwaigenbaum, L., Bryson, S., Rogers, T., Roberts, W., Brian, J., \& Szatmari, P. (2005). Behavioral manifestations of autism in the first year of life. International Journal of Developmental Neuroscience, 23 (2).

15. World Health Organization: Yemen, Annual Report 2017. 